\title{
DIAGNÓSTICOS DE ENFERMAGEM NO PÓS-OPERATÓRIO DE MASTECTOMIA
}

\author{
Nursing diagnoses after mastectomy \\ Diagnósticos de enfermería en el posoperatorio de mastectomía
}

Maria Helena Baena de Moraes Lopes ${ }^{1}$

Adriana Alves de Moura²

Sueli Raso ${ }^{3}$

Tatiana Giovanelli Vedovato ${ }^{4}$

Maria Andréia Silva Ribeiro ${ }^{5}$

\section{RESUMO}

0 objetivo desse estudo foi identificar os diagnósticos de enfermagem, no período pós-operatório de mastectomia, entre mulheres internadas em uma Unidade de Internação em Oncologia de um hospital de ensino público do interior paulista. Tratou-se de um estudo descritivo e retrospectivo, realizado em prontuários de mulheres submetidas a mastectomia. Os dados foram coletados por meio de um instrumento contendo: dados sociodemográficos, dados sobre a doença, diagnósticos de enfermagem, prescrição e evolução de enfermagem. Foram analisados 185 prontuários. Os cinco diagnósticos de enfermagem mais frequentes foram: Risco de Infecção (95,1\%), Ansiedade (48,6\%), Medo (41,6\%), Dor Aguda (14,5\%) e Mobilidade Física Prejudicada $(11,3 \%)$. Conclui-se que diagnósticos que exigem uma abordagem psicossocial para serem identificados como Distúrbio na Imagem Corporal e Angústia Espiritual foram registrados com baixa frequência, sendo mais frequentes aqueles de âmbito biomédico.

Palavras-chave: Enfermagem oncológica. Diagnóstico de enfermagem. Saúde da mulher.

\section{Abstract}

The objective of this study was to identify nursing diagnoses after mastectomy in women assisted at an oncology unit of a public teaching hospital in the interior of the state of São Paulo. This descriptive and retrospective study was performed by using medical records of women submitted to mastectomy. The data were collected by using an instrument containing: social-demographic data; the disease data, nursing diagnoses, nursing prescription and evolution. One-hundred and eightyfive medical records were analyzed. The five most frequent nursing diagnoses were as follows: Infection Risk (95.1\%), Anxiety (48.6\%), Fear (41.6\%), Acute Pain (14.5\%) and Impaired Physical Mobility (11.3\%). In conclusion, while diagnoses that require a psychosocial approach to be identified, such as Body Image Disturbance and Spiritual Anguish, were registered with low frequency, those of biomedical scope were the most frequent ones.

Keywords: Oncologic nursing. Nursing diagnosis. Women's health.

\section{Resumen}

El objetivo fue identificar los diagnósticos de enfermería después de la mastectomía en las mujeres internadas en una Unidad de Oncología en un hospital público de enseñanza en el interior del Estado de São Paulo. Fue un estudio retrospectivo, descriptivo, realizado en los registros médicos de las mujeres que se sometieron a la mastectomía. Los datos fueron recolectados por medio de un instrumento conteniendo: datos socio-demográficos, datos sobre la enfermedad, diagnósticos de enfermería, prescripción y evolución de enfermería. Se analizaron 185 historias clínicas. Los cinco diagnósticos de enfermería más frecuentes fueron: Riesgo de Infección (95,1\%), Ansiedad (48,6\%), Miedo (41,6\%), Dolor Agudo $(14,5 \%)$ y Movilidad Física Perjudicada (11,3\%). Se concluyó que los diagnósticos que requieren un enfoque psicosocial para identificación, como Disturbio de la Imagen Corporal y Angustia Espiritual se registraron con baja frecuencia, siendo más frecuentes aquellos del ámbito biomédico.

Palabras clave: Enfermería oncológica. Diagnóstico de enfermería. Salud de la mujer.

\footnotetext{
1 Enfermeira. Professora Associada da Faculdade de Enfermagem da Universidade de Campinas (UNICAMP) Campinas-SP. Brasil. E-mail: mhbaenaml@yahoo.com.br; ${ }^{2}$ Enfermeira. Especialista em Saúde Pública com ênfase em PSF e Pós-graduanda em Docência do Ensino Médio, Técnico e Superior na Área da Saúde. Enfermeira da Prefeitura Municipal de Vinhedo e Prefeitura Municipal de Jundiaí. Vinhedo-SP.Brasil. E-mail: drikinha.moura@bol.com.br; ${ }^{3}$ Enfermeira. Pós-graduanda em Docência do Ensino Médio, Técnico e Superior na Área da Saúde. Campinas-SP. Brasil. Email: su_raso2112@hotmail.com; ${ }^{4}$ Enfermeira. Doutoranda pela Faculdade de Enfermagem da Universidade de Campinas (UNICAMP). Bolsista CAPES. Campinas-SP. Brasil. tatigio@fcm.unicamp.br; ${ }^{5}$ Enfermeira. Doutoranda pela Faculdade de Enfermagem da Universidade de Campinas (UNICAMP). Enfermeira Unidade Pediatria Hospital Municipal Dr. Mario Gatti. Campinas-SP. Brasil. E-mail: mas.ribeiro@yahoo.com.br
} 


\section{INTRODUÇÃO}

Atualmente, a neoplasia mamária é o segundo tipo de câncer mais frequente no Brasil e o mais comum entre as mulheres, sendo mais prevalente na faixa etária entre 40 e 69 anos. É a maior causa de morte por câncer entre a população feminina. Em 2012, no Brasil, foram esperados 52.680 casos novos de câncer da mama, com um risco estimado de 52 casos a cada 100 mil mulheres ${ }^{1}$.

No Brasil, as taxas de mortalidade por câncer de mama continuam elevadas, muito provavelmente porque a doença ainda é diagnosticada tardiamente. $\mathrm{Na}$ população mundial, a sobrevida média após cinco anos é de $61 \%{ }^{1}$, o que demonstra a importância da doença como problema de saúde pública.

A mastectomia parcial ou radical modificada, dentre outros tratamentos existentes, é a mais indicada para o câncer de mama. Esta modalidade sofreu grandes avanços nos últimos tempos devido à evolução científica, e a técnica cirúrgica de mastectomia com reconstrução mamária imediata pode ser considerada um exemplo deste avanço².

0 período cirúrgico sempre é muito estressante, confrontando a mulher com o medo da cirurgia, morte e mutilação com a perda da mama. Este tratamento pode comprometer a mulher de maneira física, emocional e social, e a alteração da imagem corporal tem relevância para a mulher com câncer de mama ${ }^{3}$.

A unidade de internação em oncologia, na qual foi desenvolvido o presente estudo, utilizava na prática diária de trabalho os diagnósticos de enfermagem, segundo a taxonomia da North American Nursing Diagnosis Association - International (NANDA-I) ${ }^{4}$. No entanto, nenhuma avaliação com a finalidade de verificar quais eram os diagnósticos de enfermagem mais identificados em mulheres mastectomizadas foi realizado.

A questão nor teadora deste estudo foi conhecer se as enfermeiras que trabalhavam nesta unidade de internação oncológica identificavam os diagnósticos de enfermagem da esfera psicossocial e espiritual, condição que, de acordo com a literatura $3: 5$, as mulheres mastectomizadas ficam mais expostas.

Este tipo de análise poderia subsidiar futuramente atividades de educação permanente, focalizadas em melhorar a assistência prestada, além de contribuir para maior conhecimento sobre o tema, uma vez que publicações sobre ao assunto são escassas.

Em revisão da literatura, observou-se que em um estudo brasileiro ${ }^{6}$ foram identificados alguns diagnósticos como falta de padrão de exercícios, autocuidado diminuído, bem-estar psicológico prejudicado, conhecimento escolar diminuído, padrão alimentar prejudicado e ingestão de líquido diminuído em mulheres submetidas a mastectomia e que também eram participantes de um Programa de Reabilitação. Os autores ${ }^{6}$, no entanto, ressaltavam que estes diagnósticos registrados não eram exclusivos para mulheres com câncer de mama e podiam ser identificados em clientes com outras alterações de saúde.

No contexto internacional, um estudo realizado no Japão mostrou que mulheres no estágio final de câncer de mama apresentam, com frequência, dor crônica, risco de infecção e intolerância à atividade ${ }^{7}$. Já outro estudo, realizado na Espanha, detectou os diagnósticos de medo, ansiedade, déficit de autocuidado, mobilidade prejudicada, risco de baixa autoestima, enfrentamento ineficaz e complicações potenciais (dor e infecção) ${ }^{8}$.

Portanto, o objetivo do presente estudo foi verificar quais os diagnósticos de enfermagem, com ênfase na esfera psicossocial e espiritual, no período pós-operatório de mastectomia em mulheres, eram os mais identificados pelas enfermeiras que trabalhavam em uma unidade de internação oncológica.

\section{MÉTODO}

Tratou-se de estudo descritivo e retrospectivo, cujos dados foram obtidos a partir de prontuários de mulheres submetidas a mastectomia no período de 01/01/2004 a 31/12/2004, em um hospital de ensino público do interior paulista.

0 recorte temporal do estudo retrospectivo no ano de 2004 foi escolhido porque antecedeu a realização de alguns cursos de educação permanente sobre o processo de enfermagem, organizados pelo próprio serviço, a partir de 2005 , fato esse que poderia interferir nos resultados deste estudo, que visava retratar o uso rotineiro dos diagnósticos de enfermagem na unidade.

No serviço de oncologia deste hospital público especializado em saúde da mulher, a divisão de trabalho para a sistematização da assistência de enfermagem (SAE) ocorre em três turnos de plantões. Há uma enfermeira por turno: no período da manhã, ela realiza a evolução de enfermagem, identifica os diagnósticos de enfermagem e prescreve para seis pacientes. No turno da tarde e da noite são sete pacientes para cada enfermeira, totalizando, assim, 20 pacientes que devem ser avaliadas diariamente. Cada prescrição, evolução e diagnóstico de enfermagem são válidos por 24 horas. 
Os critérios de inclusão para a coleta dos dados por meio dos prontuários foram: ser do sexo feminino (a unidade atende também homens com câncer de mama), ter sido submetida a mastectomia no ano de 2004 e possuir os registros dos diagnósticos de enfermagem no prontuário. Nos casos em que houve duas internações para o mesmo procedimento, foram considerados apenas os dados da primeira internação, sendo excluídos da análise os registros de internação subsequente.

Foi obtido no serviço de centro cirúrgico acesso a uma listagem contendo os registros de mastectomia no ano de 2004. A partir desta listagem, foram identificados os prontuários e solicitados ao Serviço de Estatística Médica do hospital em questão.

Os dados foram coletados por meio de um instrumento específico, contendo o período de internação; dados sociodemográficos como: estado civil, idade, tipo de moradia, renda familiar, número de filhos e religião; realização da técnica cirúrgica com reconstrução mamária imediata, os diagnósticos de enfermagem, a prescrição dos cuidados e a evolução de enfermagem no período que sucedeu ao ato cirúrgico.

0 instrumento foi pré-testado em quarenta prontuários até chegar ao seu formato final; essa amostra foi incluída na pesquisa, após readaptação do instrumento e nova coleta.

Os dados foram inseridos em uma planilha eletrônica do Programa Microsoft Excel $® 2002$ (Microsoft, 1985-2001). Depois disso, foram calculadas as frequências absolutas e relativas dos diagnósticos de enfermagem.

Os diagnósticos de enfermagem foram agrupados de acordo com os domínios da taxionomia II da NANDA, versão 2007-20084. Essa taxonomia apresenta seu conteúdo estruturado em 13 domínios, 47 classes e 187 diagnósticos de enfermagem. Cada domínio é composto por classes e cada classe, por diagnósticos ${ }^{4}$.

Foi calculada a frequência relativa e absoluta dos diagnósticos identificados, que eram classificados de acordo com o domínio ao qual pertenciam. A análise dos dados foi realizada comparando-se os resultados com a literatura existente.

0 projeto foi aprovado pela Comissão de Pesquisa e pelo Comitê de Ética em Pesquisa da instituição de origem das autoras (Parecer $n^{0} 541 /$ 2008). Foi autorizada a dispensa do Termo de Consentimento Livre e Esclarecido por se tratar de estudo retrospectivo, que envolveu coleta de dados em prontuários. Vale salientar que a gerência do Serviço de Estatística Médica do hospital onde se realizou o estudo autorizou verbalmente uma das autoras a realizar consulta direta aos prontuários do ano de 2004.

\section{RESULTADOS}

A coleta foi realizada no período compreendido entre julho e setembro de 2008. No ano de 2004 ocorreram 250 registros de mastectomia e foram localizados todos os prontuários, sendo que, destes, $65(26 \%)$ foram excluídos: dois $(1,1 \%)$ por se tratarem de pacientes do sexo masculino, dez $(5,4 \%)$ por serem segunda intervenção cirúrgica na mama (p. ex., mastectomia da mama contralateral ou mastectomia total após quadrantectomia) e 53 $(21,2 \%)$ por não conterem o registro dos diagnósticos de enfermagem.

Foram analisados, portanto, 185 prontuários, que representaram $74 \%$ das internações por mastectomia. Cento e quarenta e oito ( $80 \%)$ tinham prescrição de enfermagem para os diagnósticos levantados, e 157 (84,9\%) continham a evolução de enfermagem realizada no período pósoperatório.

Quanto ao perfil da clientela, a faixa etária de maior frequência foi de 40 a 60 anos (45,4\%); a maioria eram de mulheres casadas ou em união estável $(32,4 \%)$, sendo que $57(30,8 \%)$ dos prontuários não continham este dado. Para $47,5 \%$, a renda familiar situava-se entre 500 e 3.000 reais, $52,4 \%$ tinham entre um e três filhos, uma parcela considerável não possuía nenhum estudo $(17,4 \%)$ ou tinha ensino fundamental incompleto $(34,1 \%)$.

Das mulheres submetidas à cirurgia de mamas no ano de $2004,6,4 \%$ realizaram mastectomia bilateral e apenas $4,8 \%$ passaram pela técnica cirúrgica com reconstrução mamária imediata; o restante das mulheres $(88,8 \%)$ realizou apenas mastectomia unilateral, sem reconstrução mamária imediata.

Foram identificados 21 diagnósticos de enfermagem, sendo que os diagnósticos de abrangência biofisiológica foram a maioria e pertenciam ao Domínio Segurança e Proteção, seguidos pelos diagnósticos do Domínio Enfrentamento/Tolerância ao Estresse. Os diagnósticos mais frequentemente registrados pelos enfermeiros foram: Risco de Infecção $(95,1 \%)$, Ansiedade $(48,6 \%)$ e Medo $(41,6 \%)$ (Tabela 1).

Os diagnósticos da esfera psicossocial constavam em 98 (53\%) dos 185 prontuários analisados, e Ansiedade e Medo foram os diagnósticos mais descritos pelos enfermeiros (Figura 1). Em $40 \%$ dos casos esses diagnósticos foram identificados concomitantemente. 
Tabela 1. Distribuição dos diagnósticos de enfermagem de mulheres mastectomizadas, de acordo com 0 domínio da taxonomia II da NANDA-I $(n=185)$

\begin{tabular}{llll}
\hline Categoria & Domínio & $\mathbf{n}$ & \% \\
\hline Risco de infecção & Segurança/Proteção & 176 & $95,1 \%$ \\
Ansiedade & Enfrentamento/Tolerância ao estresse & 90 & $48,6 \%$ \\
\hline Medo & Enfrentamento/Tolerância ao estresse & 77 & $41,6 \%$ \\
Dor aguda & Conforto & 27 & 14,5 \\
\hline Mobilidade física prejudicada & Atividade/Repouso & 21 & $11,3 \%$ \\
Integridade da pele prejudicada & Segurança/Proteção & 15 & $8,1 \%$ \\
*Conforto alterado & - & 12 & $6,4 \%$ \\
Risco de lesão & Segurança/Proteção & 11 & $5,9 \%$ \\
\hline Proteção ineficaz & Segurança/Proteção & 8 & $4,3 \%$ \\
Padrão respiratório ineficaz & Eliminação/Troca & 8 & $4,3 \%$ \\
\hline Pesar & Enfrentamento/Tolerância ao estresse & 6 & $3,2 \%$ \\
Distúrbio na imagem corporal & Autopercepção & 3 & $1,6 \%$ \\
Risco de nutrição desequilibrada: & Nutrição & 3 & $1,6 \%$ \\
\hline Eliminação urinária prejudicada & Eliminação/Troca & 2 & $1 \%$ \\
\hline Risco de constipação & Eliminação/Troca & 2 & $1 \%$ \\
Déficit no autocuidado & Eliminação/Troca & 2 & $1 \%$ \\
Confusão aguda & Percepção/Cognição & 2 & $1 \%$ \\
Náusea & Conforto & 2 & $1 \%$ \\
Controle ineficaz do regime terapêutico & Promoção da saúde & 1 & $0,5 \%$ \\
Angústia espiritual & Principios da vida & $0,5 \%$ \\
\hline
\end{tabular}

* Não está incluído na taxonomia II da NANDA, mas é referido na literatura

Figura 1. Diagnósticos psicossociais identificados no pós-operatório de mulheres mastectomizadas $(n=98)$

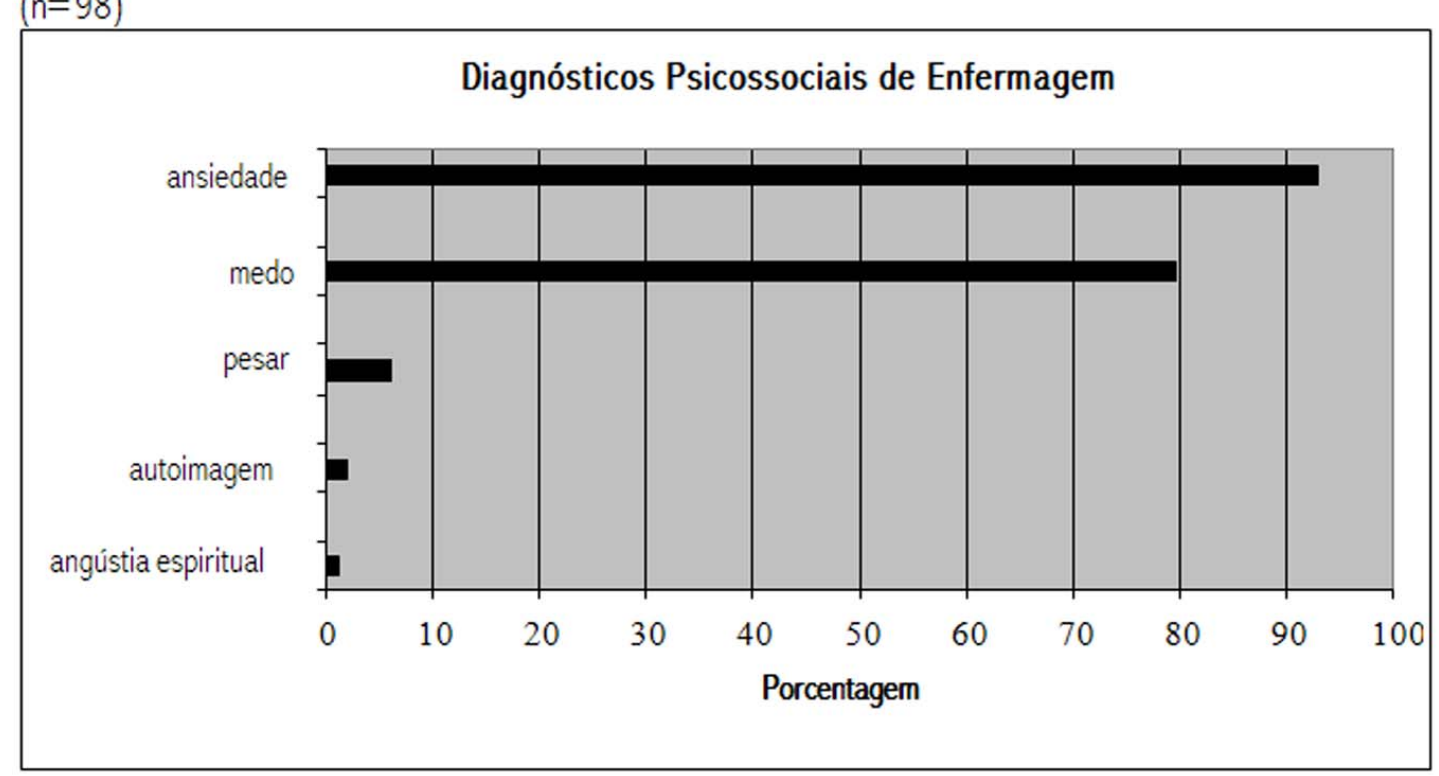

\section{DISCUSSÃO}

No presente estudo, as mulheres na faixa etária entre 40 e 60 anos representaram 45,4\% da amostra estudada, de acordo com a literatura que mostra que a neoplasia mamária é mais prevalente na faixa etária de 40 a 69 anos ${ }^{1}$.

As enfermeiras registraram nos prontuários, com maior frequência, diagnósticos dos domínios Segurança/ Proteção e Enfrentamento/Tolerância ao Estresse, destacando-se o diagnóstico Risco de Infecção. Esse resultado coincide com os achados de outros estudos que 


\section{Diagnóstico de enfermagem pós-mastectomia}

Lopes MHBM, Moura AA, Raso S, Vedovato TG, Ribeiro MAS

mostram ser este o diagnóstico mais comum em pacientes submetidos a procedimentos cirúrgicos e/ou pacientes oncológicos $^{7-9}$.

Um estudo retrospectivo, realizado a partir de dados coletados em prontuários de uma unidade de internação em oncologia, indicou que Risco para Infecção encontrava-se entre os cinco diagnósticos de enfermagem mais frequentes ${ }^{9}$ e considerou que $100 \%$ das pacientes que iriam ser submetidas a cirurgia teriam esse diagnóstico. Ressalta-se que este estudo ${ }^{9}$ foi desenvolvido na mesma unidade de internação onde foram coletados os presentes dados, mas, naquele momento, ainda não se utilizava a taxonomia NANDA-I e os diagnósticos foram determinados a partir das características definidoras ou fatores de risco registrados no histórico de enfermagem.

A validação dos diagnósticos que envolvem respostas subjetivas é um processo árduo e tem sido objeto de estudo há algum tempo. Em um estudo sobre a validação dos diagnósticos Ansiedade e Medo ${ }^{10}$, ressaltou-se a presença de características definidoras e fatores relacionados similares para esses dois diagnósticos. De fato, eles compartilham 20 características definidoras idênticas ou similares na versão 2007-2008 da taxionomia da NANDA-I ${ }^{4}$, o que torna difícil a diferenciação entre essas reações humanas.

Assim, era esperado que, no presente estudo, os diagnósticos da esfera psicossocial Ansiedade e Medo fossem identificados de forma concomitante; esse fato foi comumente observado, uma vez que as enfermeiras os registram, na maioria das vezes, sob a forma "Ansiedade/Medo".

0 diagnóstico Conforto Alterado, sugerido por Carpenito ${ }^{11}$, foi identificado em $6,4 \%$ dos prontuários. Ressalta-se que este diagnóstico não faz parte da taxonomia da NANDA-I, mas é comum encontrá-lo na prática de enfermagem ${ }^{12}$.

Diagnósticos como Distúrbio na Imagem Corporal, Pesar e Angústia Espiritual foram poucas vezes registrados pelas enfermeiras que atuam nesta unidade de internação oncológica; no entanto, a ocorrência de mutilação de uma parte do corpo da mulher (mama) e o surgimento de sentimentos negativos são comuns neste tipo de clientela ${ }^{12}$.

A mulher, ao receber um diagnóstico de câncer de mama, vivencia vários lutos internos, de acordo com a intensidade do sofrimento ${ }^{13}$. A mastectomia costuma causar profundos impactos psicológicos, abalando sua autoestima. Quando associada à quimioterapia, esse impacto aumenta em função dos efeitos colaterais decorrentes, especialmente a alopecia, e a mulher apresenta respostas negativas refletidas pelo medo, depressão, angústia, tristeza, distúrbio da imagem corporal e pesar antecipado ${ }^{14}$.

A técnica de mastectomia com reconstrução mamária é uma modalidade cirúrgica existente que contribui em vários aspectos na melhora os conflitos internos da mulher, bem como da sua qualidade de vida após a mastectomia, porém nem todas têm a possibilidade usufrui-la ${ }^{2}$. No presente estudo, apenas 4,8\% passaram por técnica de mastectomia com reconstrução imediata, mas apesar disso somente $1,6 \%$ das mulheres receberam o diagnóstico Distúrbio na Imagem Corporal.

Considerando o baixo percentual de mastectomia com reconstrução mamária imediata e o possível impacto que isto poderia ter sobre a qualidade de vida da mulher e sua autoimagem, era esperado que a frequência do diagnóstico Distúrbio na Imagem Corporal fosse elevada, o que não ocorreu. Isso indica que, provavelmente, não houve identificação, por parte das enfermeiras, das características definidoras destes diagnósticos. Outra possibilidade seria a qualidade da assistência prestada ou características pessoais da população em estudo, que contribuiriam para a baixa frequência deste diagnóstico. Esses são aspectos que requerem futuras investigações.

Assim, este resultado diverge da literatura dentre os distúrbios psicossociais apresentados pela mulher mastectomizada, pois o mais comum é o da imagem corporal $^{3,5,13,15}$.

Uma doença como o câncer de mama, que de fato é debilitante e requer tratamento prolongado, pode levar a alterações no processo familiar, considerandose que a clientela atendida é predominantemente feminina e responsável pela manutenção do lar como donas de casa e/ou como trabalhadoras ${ }^{9}$. Além disso, a mastectomia leva a restrição de algumas atividades domésticas e cuidados pessoais como erguer pesos, serviços de jardinagem, depilação axilar, cortar as unhas, lidar com objetos cortantes como facas, tesouras, entre outros. De fato, muitas mudanças surgem na vida da mulher em função do câncer de mama, levando a sentimentos que podem modificar a sua imagem corporal, autoestima e relacionamento social ${ }^{16}$.

Embora nenhum diagnóstico relacionado à esfera social tenha sido identificado pelas enfermeiras durante o período de internação, devido ao risco de ocorrência, esses aspectos devem ser investigados e discutidos com a mulher e sua família antes da alta, a fim de prevenir ou minimizar as consequências. Assim, uma investigação dos diagnósticos do Domínio Autopercepção deveria ser incluída no plano de cuidados pós-operatório da mulher mastectomizada, o que parece não ter sido realizado na unidade hospitalar oncológica em análise.

Os resultados encontrados sugerem, portanto, que a realidade empírica nem sempre caminha em consonância com a científica e que existem dificuldades na aplicabilidade desses diagnósticos de enfermagem em oncologia mamária feminina.

Ao enfermeiro incube-lhe evidenciar respostas 
humanas adequadamente e incluir no seu planejamento de cuidados medidas terapêuticas específicas. 0 uso de instrumentos de medida como escalas psicométricas seria muito útil não apenas para identificar diagnósticos da esfera psicossocial, mas também para avaliar o efeito das intervenções de enfermagem.

Qualquer enfermeiro ao formular um diagnóstico depara-se com a difícil tarefa de julgar qual seria aquele que melhor representa um determinado conjunto de características definidoras, bem como a necessidade de considerar cuidadosamente as manifestações do paciente diante das características definidoras de cada diagnóstico ${ }^{15}$. Além de ter familiaridade com o processo de enfermagem, precisa explorar conceitualmente os diagnósticos potencialmente frequentes na sua prática clínica; isso facilita a eficácia do processo e diminui os erros diagnósticos.

Uma das funções do enfermeiro é assegurar a qualidade de vida da mulher mastectomizada por meio de ações educativas, sendo tão relevantes quanto os cuidados clínicos. Assim, a experiência da mastectomia pode ser menos traumática para as mulheres com câncer de mama se houver pleno empenho dos enfermeiros ao considerarem os aspectos psicossociais ${ }^{16}$, a fim de estabelecerem diagnósticos de enfermagem que embasam essas ações.

No período pós-operatório de mastectomia, a assistência de enfermagem deve direcionar-se para os cuidados domiciliares, incentivando o autocuidado, avaliando o grau de dependência e solicitando encaminhamento aos grupos de apoio interdisciplinares indicados ${ }^{17}$. Assim a identificação adequada dessas necessidades é essencial.

0 profissional enfermeiro é o integrante da equipe multidisciplinar que permanece maior tempo em contato com mulher portadora de câncer mama, permitindo, dessa forma, atuação integral, sustentada pelo diagnóstico de enfermagem, seja na qualidade de prestador de cuidados ou de educador em saúde. Essas ações valorizam sua atuação e reforçam a importância do uso do processo de enfermagem, de garantir a sistematização das ações e o seu adequado registro, contribuindo para a sua autonomia profissional.

As limitações encontradas no decorrer dessa pesquisa foram relacionadas ao fato de serem dados retrospectivos, de prontuários. Como há escassez de pesquisas, na literatura nacional e internacional, referentes ao processo de enfermagem, especificamente envolvendo mulheres com câncer de mama e mastectomizadas, recomenda-se que sejam desenvolvidos estudos prospectivos, a fim de identificar prioridades e desenvolver métodos de avaliação sensíveis aos efeitos das intervenções de enfermagem propostas com base nos diagnósticos identificados.

\section{CONCLUSÃO}

Os diagnósticos de enfermagem mais identificados pelas enfermeiras que atuavam em uma unidade de internação oncológica foram os que pertenciam aos domínios Segurança/ Proteção e Enfrentamento/Tolerância ao Estresse, destacandose o diagnóstico Risco de Infecção, que está de acordo com o esperado.

No entanto, alguns diagnósticos da esfera psicossocial que exigem do enfermeiro uma abordagem mais aprofundada para sua identificação foram registrados com baixa frequência.

Os diagnósticos de enfermagem nas esferas psicossociais mais encontrados foram a Ansiedade e Medo, por possuírem características definidoras semelhantes e pelo perfil da população estudada. No entanto, os diagnósticos que exigem do enfermeiro uma abordagem mais criteriosa na elaboração foram registrados com baixa frequência.

Diante do exposto, torna-se evidente a importância de a enfermagem oncológica respaldar-se também nos diagnósticos psicossocias na elaboração do seu plano de cuidados às mulheres mastectomizadas, assegurando a melhoria da assistência prestada.

Como validar os diagnósticos de enfermagem em diferentes contextos e populações representa um desafio para a enfermagem, faz-se necessário desenvolver mais estudos nesta direção.

\section{REFERÊNCIAS}

1.Instituto Nacional de Câncer José Alencar Gomes da Silva. Coordenação de Prevenção e Vigilância de Câncer. Estimativa 2012: incidência de câncer no Brasil. Rio de Janeiro: Inca; 2011.

2.Gregório TCR, Sbalchiero JC, Leal PRA. Exame histopatológico das cicatrizes de mastectomia nas reconstruções tardias de mama: existe relevância oncológica? Rev. bras cancerol. 2007; 53(4): 421-4.

3.Camargo TC, Souza IEO. Atenção à mulher mastectomizada: discutindo os aspectos ônticos e a dimensão ontológica da atuação da enfermeira no Hospital do Câncer III. Rev. Latino-Am. Enfermagem. 2003 Set/Out; 11(5): 614-21.

4.North American Nursing Diagnosis Association. Diagnósticos de Enfermagem da NANDA: definições e classificação 2007-2008. Porto Alegre: Artmed; 2008. 
5.Vieira CP, Lopes MHBM, Shimo AKK. Sentimentos e experiências na vida das mulheres com câncer de mama. Rev. Esc. Enferm. USP. 2007; 41(2):311-6.

6.Primo CC, Leite FMC, Amorim MHC, Sipioni RM, Santos SH. Uso da classificação internacional para as práticas de enfermagem na assistência a mulheres mastectomizadas. Acta paul. enferm. 2010; 23(6):803-10.

7.Ogasawara C, Hasegawa T, Kume Y, Takahashi I, Katayama Y, Furuhashi Y, Yamamoto Y, Okazaki S, Tanabe M. Nursing diagnoses and interventions of Japanese patients with end-stage breast cancer admitted for different care purposes. Int J Nurs Terminol Classif. 2005 Jul; 16(3-4):54-64.

8.Peinado-Barraso MC, Cabrerizo-Cordero MR, Granados-Matute AE, Contreras-Fariñas R. Case report: coordination of the care provided to patients with breast cancer. Enferm Clin. 2008 Sept/Oct;18(5):273-7.

9.Lopes, RAM, Macedo DD, Lopes MHBM. Diagnósticos de enfermagem mais frequentes em uma unidade de internação de oncologia. Rev. LatinoAm. Enfermagem. 1997; 7(5): 77-83.

10.Whitley GG, Tousman AS. A multivariate approach for validation of anxiety and fear. Nurs. diagn. 1996; 7(3): 116-24.

11.Carpenito L. Compreensão do processo de enfermagem: mapeamento de conceitos planejamento do cuidado para estudantes. Porto Alegre: Artmed; 2007.

12.Gouveia, HG, Lopes MHBM. Diagnósticos de enfermagem e problemas colaborativos mais comuns na gestação de risco. Rev. Latino-Am. Enfermagem. 2004 Mar/Abr; 12(2): 175-82.

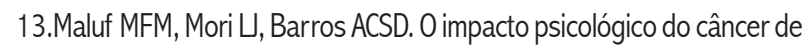
mama. Rev. bras. cancerol. 2005; 51(2): 149-54.

14.Melo EM, Araujo TL, Oliveira TC, Almeida DT. Mulher mastectomizada em tratamento quimioterápico: um estudo dos comportamentos na perspectiva do modelo adaptativo de Roy. Rev. bras. cancerol. 2002; 48(1): 21-8.

15.Braga CG, Cruz DALM. Sentimento de impotência: diferenciação de outros diagnósticos e conceitos. Rev. Esc. Enferm. USP. 2005; 39(3): 350-7.

16.Moura FMJSP, Silva MG, Oliveira SC, Moura LSP. Os sentimentos das mulheres pós-mastectomizadas. Esc. Anna Nery Rev. Enferm. 2010 Jul/ Set; 14(3): 477-84

17.Normas e recomendações do Ministério da Saúde no controle de câncer de mama (BR). Rev. bras. cancerol. 2004; 50(2): 77-90. 